\title{
Global Hydrocarbon Energy Potential of Euro-Asia or Solution of Navier-Stokes Equation for Deformed Micro-Structure of the Earth's Porous Space
}

\author{
Vyacheslav I. Popkov ${ }^{1,3}$, Vladimir I. Astafiev ${ }^{1}$, Alexander M. Shterenberg ${ }^{2}$, Ilus G. Khamitov ${ }^{3}$, Vladimir A. \\ Kolesnikov $^{3} \&$ Alevtina V. Popkova ${ }^{3}$ \\ ${ }^{1}$ Oil \& Gas Field Development \& Operation Dept., Samara State Technical University, Samara, Russia \\ ${ }^{2}$ General Phys. \& Phys. of Oil \& Gas Industry Dept., Samara State Technical University, Samara, Russia \\ ${ }^{3}$ Dept. On Heavy Oil \& Natural Bitumen, OOO SamaraNIPIneft", Samara, Russia \\ Correspondence: Vyacheslav I. Popkov, Oil \& Gas Field Development \& Operation Dept., Samara State Technical \\ University \& Dept. on Heavy Oil \& Natural Bitumen, OOO SamaraNIPIneft, Samara, Russia. Tel: \\ 7-846-205-8653. E-mail: PopkovVI@samnipineft.ru
}

\author{
Received: April 1, $2015 \quad$ Accepted: April 15, $2015 \quad$ Online Published: August 30, 2015 \\ doi:10.5539/mas.v9n9p307 URL: http://dx.doi.org/10.5539/mas.v9n9p307
}

\begin{abstract}
The authors present the hydro-geological basin-type model of global energetics with hydro-dynamic potential showing the interaction of inland platforms of the Earth. The authors propose the new cluster-type approach towards the asymmetric multi-scaled modeling at the basis of integral solution within the quadrature of Navier-Stokes equation for deformed micro-structure of Earth's porous space. We have studied the effect of poor local tectonic faults upon the arrangement of hydrocarbon traps in lithosphere, shale tight fields, that is based upon the interconnected methods in geophysics, hydro-geology and geo-mechanics, tectono-physics and structural geology. The authors also present the scientific concept of new principles to increase the level of geo-informatics while searching exploring and developing the hydro-carbon reserves, geo-physical studies of seismic emissions, micro-seismic and seismology of mega-scales in geological ages of stresses with transition to predictable man-induced time for geological/technological events. Basing upon the conjugation of Navier-Stokes equation and due consideration of deforming relaxation we have built the energy self-arranged model of Earth's lithosphere de-fluidization, have found inherent energy delayed spectra for the dynamic contacts of stratified structure. We have obtained the energy conjugated solution of micro-structure porous media interaction for hydro-geological decompression of subduction channel in seabed and saturation of generalized Middle Oceanic Ridge with self-organization of classic sedimentological hydro-carbon traps and complexly organized shale reservoirs with compacted depositions of Allochthon structures in Domanik and Bazhenov formations. The authors present the energy stable mean filtration profile as per Darcy, conjugated with stress-deformed status of porous geo-media micro-structure at various boundary conditions: closed-type or capillary jammed boundaries of filtration blocks and free-type - with phase filtration front and diffusive displacement of fluids by saturated porous media and smart technologies of multi-stage hydro-fracture in dense shale reservoirs. Analytically, in quadrature, there is the dissipation velocity, potential and kinetic energy potentials of mean outflow and shear layer of filtration dissipative structure, like a generalized model of viscous elasticity that depends upon the wave number, phase velocity, thickness of the layer and geo-mechanical parameters that reflect the geo-media heterogeneity.
\end{abstract}

Keywords: middle oceanic ridge, Earth's structure, Allochthons, global energetic, geo-informatics, seismic emission

\section{Introduction}

The development of the scientific ideas in XXI century has started with the solution of the fundamental issues of the previous century. The arrangement of a phenomenological model of organization and interaction of complexly organized large-scaled structures with the solution of Navier-Stokes equation is the most important objective that is still not-solved starting from the previous century. May the global mathematics resolve the system problems of the geology (Astafiev et al 2014, Popkov et al 2012-2014), conjugated with geo-physics and 
global economics? The problem initiated by Vernadskiy V. I. (Vernadskiy 2001) still has no answer. The complexity in methodical substantiation of the problem contains the fact that many branches of science, like physical chemistry, nuclear physics, quantum transportation, are using asymmetric interaction models, and some of them, like hydro-geology. Hydro-dynamics or porous filtration - symmetrical ones (Nikolaevskiy 1996-2010), Garagash et al 2009, Molokovich 2006, Shifrin et al 2010, Sheretov et al 2009, Dmitriev et al 2003, Radchenko et al 2000, Burgers 1974). However, the fundamental problem of the global energetic, Earth's ecology is not only the symmetrical simplification of the problem with geological risks, but this is the search of a powerful fundamental solution for mass transfer, that is energetically conjugated with balanced adiabatic micro-structure. Moreover, the structure of a microscopic droplet turned out to be limitless as the same with atom, and physical and mathematical description of its energetic is still not possible. Niels Bohr once said, that "the problem turned out to be more important than its solution, the solution has become out of date and the problem still remains the same".

The solution of the considered problem boiled down to the objective in arranging the basinal-type model for the energetic structure of the continents and oceans that is invariantly conjugated with the micro-structure of Earth's lithosphere de-fluidization. The boundaries of the continent are not the only things that unite the far away areas of the territory having both, Iceland and Sakhalin. Besides, they are also formed by the global subduction of the oceans, by the deep geo-dynamics of Earth's energy potential genesis, migration of "life energy" to the continents. Unified Eurasian energy drain of giant planetary oceans' flow is the Middle Oceanic Ridge. i.e. The Urals. Neo-tectonics of the Cis-Ural region goes to deep micro-structural geo-chemical transformations in ancient oceanic migration compacted sediments by decomposed foundation trap-rocks - in oil-saturated matrix rock of Domanik in the Volga region and Bazhenov formations in Western Siberia, with further arrangement of sedimentary classic porous traps with hydro-carbons $(\mathrm{HC})$. There is the rock return process in structures of foreign migration of Atlantic Ocean to the Cis-Ural region at the western slopes of the Urals, and Arctic and Pacific Oceans - to Western and Eastern Siberia. Following the studies performed by the Geological Institute at Bashkir Academy of Sciences (Kamaletdinov et al 1981) the ridge of the Urals is not presented by volcanic rock but is arranged by foreign deeply-migrated micro-structure of crushed Allochthons. Basinal-type modeling has proved that Sic-Urals area had not produced the excessive amount of sediments so as to fill-in all the sedimentary traps with HC. The major part of the Volga area sedimentary rock, for example, has been moved from the Baltic Platform, areas of the Caspian and Mediterranean seas, Figure 1. The Volga area Domaniks as per the recent core studies are presented by Allochthonic micro-structure of the migration-sheared layer. In the sedimental rock of the Southern Urals and Caspian Sea area the important role is played by the Indian Ocean and this is verified by the structural maps of the stresses in deterministic moments of the region as built by the Institute of Physics at Russia Academy of Sciences (RAS) (Mukhamediev et al 2004).
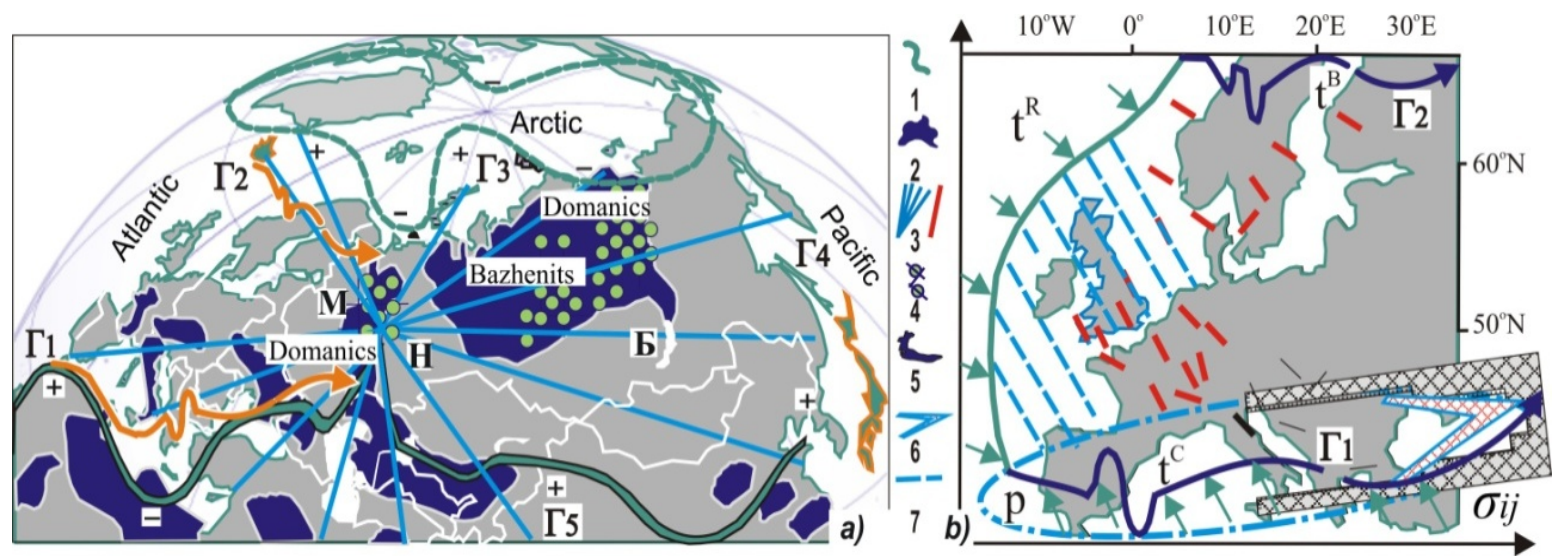

Figure 1(a). De-fluidization of the Earth at (b) spreading: by decompression 1 and compression 2 for traps 3, Allochthons 4, sediment basins 5, migration 6 and saturation 7 of generalized $\mathrm{t}^{\mathrm{R}}$ in Middle Oceanic Ridge $\mathrm{H}$ with contracts loading-out for Mediterranean $\Gamma_{1}$ and Baltic $\Gamma_{2}$ seas, Arctic $\Gamma_{3}$, Pacific $\Gamma_{4}$, Indian $\Gamma_{5}$ oceans and boundaries of African and Euro-Asian $\mathrm{t}^{\mathrm{C}}$, Baltic $\mathrm{t}^{\mathrm{B}}$, Moscow M, Baikal platforms $\mathrm{b},+/-$ up having (for oil)/

down-throwing (for gas) 
The science of Earth's dynamic dissipative structures, of spatially distributed reactive-diffusional multi-scaled systems has recently become a powerful scientific area with well-developed experimental and theoretical base (Sheretov 2009, Dmitriev et al 2003, Kamaletdinov et al 1981, Mukhamediev et al 2004, Weinberg 2009). The problems with non-elastic deformation of porous structure, destruction of continuous medium (Garagash et al 2009, Burgers 1974, Bykova 2010, Ayachi et al 2013, Popkov et al 2014, Jacobs 2014, Gueguen 2004, Mironova 2013), non-stationary filtration, smart technologies in HC development, Smart Wells (Popkov et al 2013) present a specific area of interest for the scientific community, both in this country and abroad. The problem has become especially acute while considering the concept of oil genesis (Khain et al 2005, Dmitrievskiy et al 2003, An 2009), models of dynamic core wave-guides, "shale revolution" and development of Arctic off-shore areas, Bazhenov formation and Allochthonic "foreign" structures with migration of oil-bearing Domanik in the Volga-Urals and Eastern Siberia regions. The ancient Allochthons of remote Domaniks Atlantic and Pacific Oceans, Bazhenov formations- the nearest, Arctic Ocean differ in complexly structured multi-scaled tangential diffusively crushed shale structure of dense reservoirs at the final stage of oil-bearing sediment rock formation.

At present there is no global energetic basinal-type model of the Earth, its ecological safety and geological risks. The ancient civilization of the Earth, the basement of which was found at the bottom of the Mediterranean Sea region or near Japan or the pyramids of the Indian off-shore are clear evidence of this case. Tangentially crushed by the geological ages their traces should be looked for in the deep Allochthonic migration flows of decompressed traps of crystalline foundation, in oil-bearing rock depositions of the Caucasus, Caspian Sea, Sic-Urals and Siberia, in HC porous traps, in dissipative structures of dense Kerogen and dissipated geo-spheres of the Earth. The presented model of integrated energy solution of the problem at the basis of the analytical solution of N.-Stokes equ's for the rigidly conjugated porous space of Earth's de-fluidization, movement of deep-type mantle energy and its transformation into potential energy of life is the highest form of self-organization in energy structure. The objective of this paper is the reproduction of conjugated structure as the solution "of the challenge of the century" as well as geo-dynamic analysis of the solution through numerous multi-year case studies and mathematical modeling with 4D Assisted History Matching.
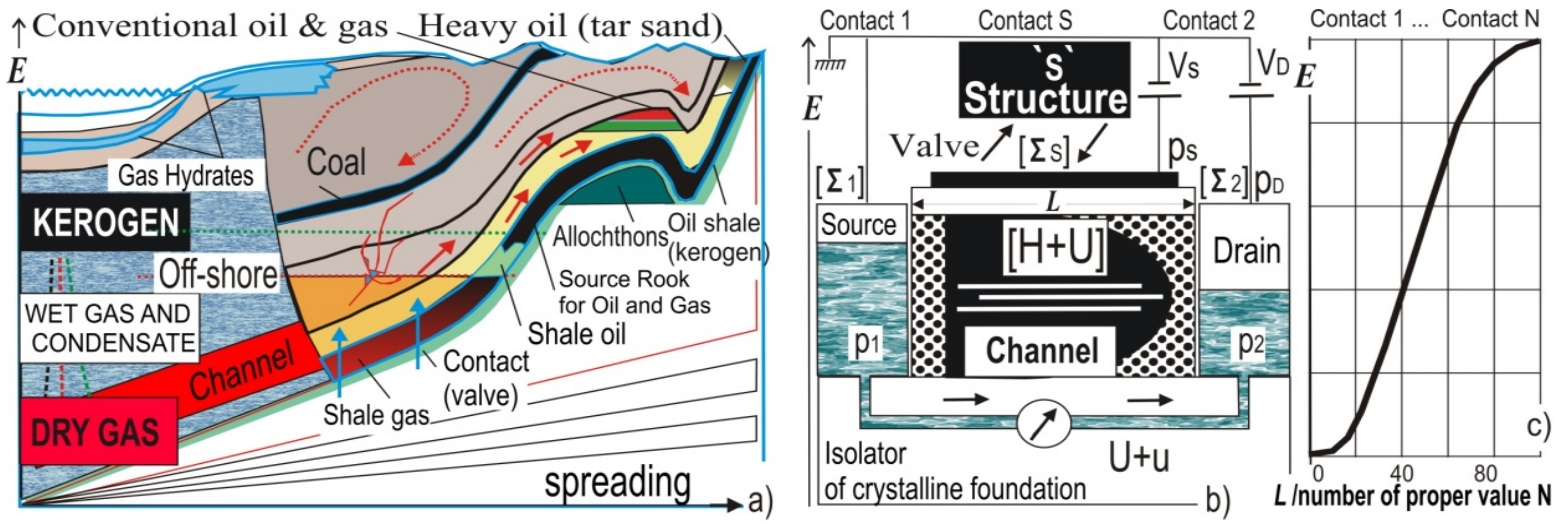

Figure 2(a). Coordination of porous structure transport conductivity with (b) conductance of transistor and (c) Free energetic potential of deformation

Legend: I-flow, V-stress, E-energy, G-conductance/conductivity, H+U- potential for channel widening, $\Sigma$-eigen energetic function (with delay), $\Sigma$ s-function of structure dissipating, H-Hamiltonian, U-self-coordinated potenti$\mathrm{al}, \mathrm{p}_{\mathrm{S}}, \mathrm{p}_{\mathrm{D}}$ - pressure in matrix and reservoir

The problem with energetic modeling of the lithosphere includes the provision of new experimental and field geo-physical and geological data that are not lined with the approved phenomena-type models and laws. The experiments and the theoretical studies have proved the existence of the surface layers on the Earth that possess their own internal structure. Dmitrievskiy A. N. has developed a concept of vortex-type Earth's geo-dynamics and block-type organization of the lithosphere. Looking at the physical models of the Earth the development of torsion field, spirals and vortex is correlated with Bembel's R. M. solitons that reflect the first organizational level in the system of physical fields and geological processes. Sokolov B. A. \& Ablya E. A. (1999) have proposed fluid/dynamic model of oil and gas origin. The model is based upon the properties of sedimentary rock 
in the process of lithogenesis to depart into zones of compaction and de-compaction. The same abnormal filtration of $\mathrm{HC}$ cannot be described by parabolic equ's like thermal conductivity, and this required systematic integrated conjugation equ's for mass and energy transfer (Demyanov et al 2004, Kulesh et al 2005, Akbarov et al 2011, Berdnikov et al 2014, Astafiev et al 2015). Modification of differential equ's (DE) in view on their instant micro-structure is described in papers of Koser E. (1903), Sedov L. I., Nikolaevskiy V. N. (2010). On a system-type level this intellectual method to increase the geo-information in search, exploration and development of oil and gas fields, the study of man-made process effects upon biosphere, Palae- and Neo-tectonic evolution of the lithosphere is the further development of the ideas by Vernadskiy.

There are wave-type processes in the spherical Earth's mantle that reflect the transfer, accumulation and transformation of energy. These are electro-magnetic, gravitational, thermal, elastic/seismic, phase transitions and many other types. Between the mantles there exist the reflecting boundaries that arrange energetic barriers between them. While forming the elements of self-wave system there appear the cells capable to keep their own energy. Concentrating in the cells the energy in resonant mode interacts with adjacent cells and "over-emitting" increase the amplitude and scale of interaction potential (Volodin 1999).

The basinal - type model of Earth's defluidization (at the basis of N.-Stokes equ's) has both particulate and wave solutions. The system integration of an ecological safety solution is possible at the basis of fundamental solution of N.-Stokes equ's, synchronized model of critical risks, break-through concepts of phenomenological evolutionary ideas and development of economy. This is evidently seen today when Russia has announced the process of Arctic off-shore area development and the USA- the process of "shale revolution" simultaneously with the implementation of intellectual sanctions and localization policy in the area of energetics. The conjugation of N.-Stokes equ's with micro-structure of deformed porous space will enable us to solve the similar tasks with the growth in geo-information for global energy modeling.

The objectives with fluid filtration deal with the fundamental issues of physics from the nature of micro-world friction at the atomic-molecular levels of phase and surface layers up to micro-world with the emerging of irreversibility in the initially inverted by time laws and presentation of dissipative stimulus. Developing the variation principle of elasticity, Sedov L. I. noted that the velocity of flow having both maximum and minimum values is organized appropriate contacts of the reservoirs. Christianovich S. A. (1933) has performed the dynamical analysis with various in structure river hydraulics and with dissipative filtration. Figure 3a presents the structural surface of $\mathrm{HC}$ oil and gas saturated pool with complicated structure and surface $\Sigma \mathrm{s}$ of the contacts: strengthening $\Gamma_{\mathrm{T}}$, decompressed $\Gamma_{\mathrm{B}}$, compaction $\Gamma_{\mathrm{z}}$, plastic decompression $\Gamma_{\mathrm{\phi}}$, facies boundaries of sedimentation repeatability, gas/oil (GOR) $\Gamma_{\phi}$ and water/oil (WOC) $\Gamma_{\mathrm{o}}$ contacts. The process to develop HC using horizontal $(\mathrm{HW})$ and vertical (VW) wells contains the respective un-loading pressure trajectories $\Gamma_{\mathrm{o}}$ and $\Gamma_{\phi}$ for adjacent decompressed structures with elastic-fractured, elastic- and compacted-fractured double porous media in layered stratified pools. The fields with this HC organization structures were discovered and are operated in many Euro-Asian areas with oil and gas production: Samarskaya region, Bashkortostan, Western and Eastern Siberia, Peri-Caspian region, Vietnam.

Reactive diffusion dissipative structures (Christianovich 2000, Vanag 2008) with porous space were discovered at facies boundaries of the traps, inflow and outflow contacts, Figure 2. Comparing Ohm and Darcy laws by analogy enables us to coordinate the dynamic permeability of porous structure contacts with quantum conductivity transport of field transistor (Datta 2009). For such a transistor the contact of the conductor with matrix is represented by ballistic, tunnel and resonant structures and for filtration by Darcy- by a single symmetrical ballistic structure of porous space having elliptical or parabolic type. The deformed de-compressed micro-structure of the filtration is presented by tunnel slot-type conductivity in single-axle fracturing, and for compacted matrix of viscous skeleton- by resonance type. The structural development of filtration in films, droplets and lamellae may be represented by the momentum N.-Stokes equ's, that allows pulse solution of convective diffusion contact between conductor and matrix (model, Figure 3c), and cannot be described by the parabolic equ's of thermal conductivity type (IPR curve). The similar numerical N.-Stokes equ's for the energy structure in porous channel in electro-magnetic field of the Earth have the configuration of a stagnant interferential wave for resonant interference micro-structural deformation and know-type conjugations of critical filtration surfaces in various geo-physical fields.

Pressure Indicating Diagram (ID) showing the dependence of rate Q versus pressure p inflow chokes $\varnothing(2-8)$, (Figure 3b), of experimental results at Well Hydro-Dynamic Survey in complexly-organized reservoirs demonstrate severe non-linear characters of permeability $k$ function. We specify the structures with de-compaction, strengthening and resonant-strengthening that are described by elliptical, parabolic and hyperbolic equ's respectively. Evolutionary interactions of parabolic structures with wavy parts of non-linear 
diffusion were studied by Burgers J. M. (1974) at momentum equ's to preserve the number of movements. The equ's of N.-Stokes enables to describe the integrated/ conjugated interaction of 4D (+time) energy transfer and the development of permeability (Jacobs 2014, Gueguen et al 2004) and to approach the solution of Earth's de-fluidization that is based upon the concept of spreading for de-compacted traps in crystalline basement.
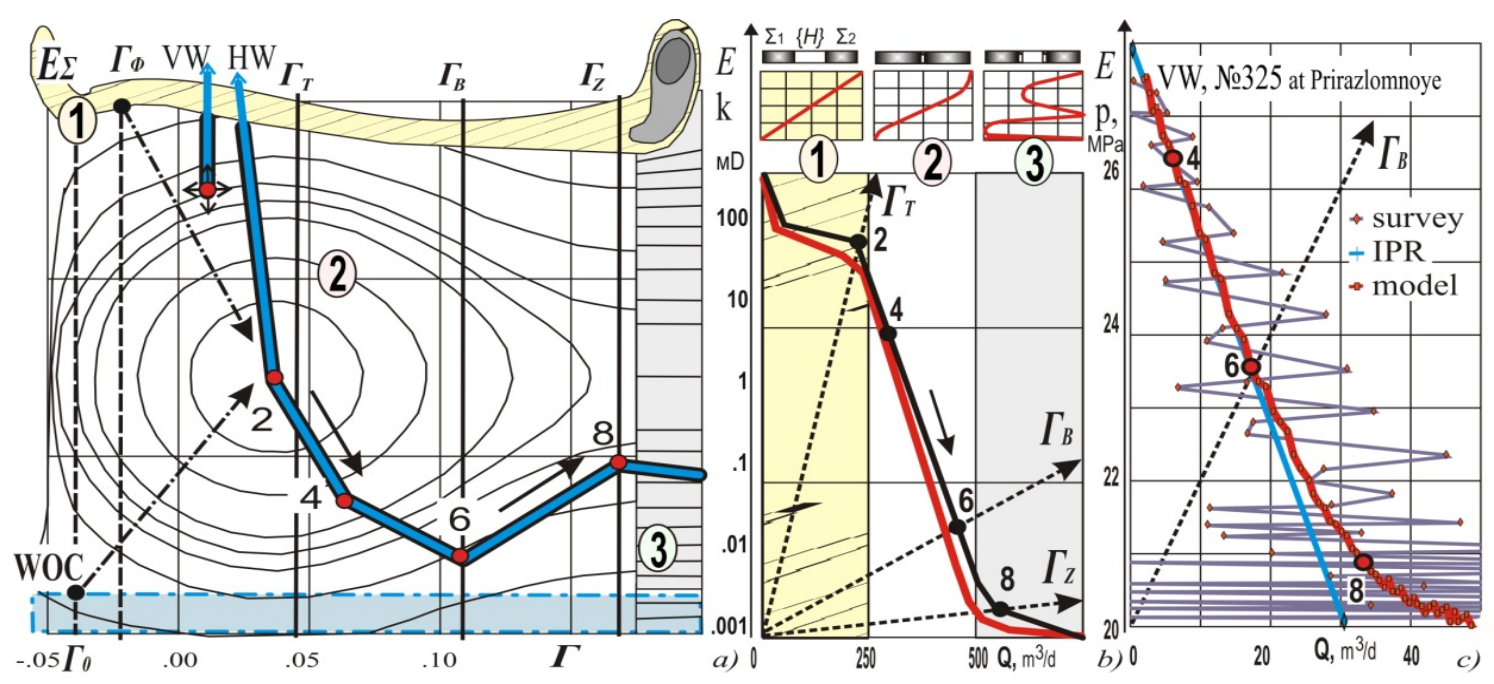

Figure 3(a). Surface free energy $E_{\Sigma}$ potential relieving $\Sigma$ by strengthening of elliptical $\Gamma_{\mathrm{T}}$, parabolic $\Gamma_{\mathrm{B}}$, hyperbolic $\Gamma_{z}$, tunnel-type $\Gamma_{\phi}$ structures at $\Gamma$, WOC $\Gamma_{0}$, GOC $\Gamma_{\Phi}$; (b) reservoir IPR curve for conductivity/ permeability of tunnel-type 1, ballistic 2, resonant 3 micro-structures, (c) ID well № 325

Analysis of evaluation results with theoretical and experimental approaches status towards the basic problems with condensed media allows to discover actual trends in developing the methodology in survey of macroscopic properties of actual natural and man-made systems, the phenomena of which are defined by their physics/chemistry, and are directly linked with practical aspects in unifying the existing variety in methods of their study. The clear realization of a principle with dynamic correspondence in physics/chemistry of the condensed status to macroscopic properties of the substance through isotopic molecular systems provides the possibility in their unification. The development of theoretical base for the established principle of electro-dynamic correspondence, in line with methodical base of electro-mechanical instrument practical application defines the significant widening of analytical possibilities to study and understand the phenomenon of condensed status.

At present there are no substantiated and intensive development of conceptual approaches towards the realization of these possibilities "at the boundary" between the methods of mechanical continuous medium, physics/chemistry and macroscopic dynamics for continuous medium. The phenomenological principles, considered at traditional basis, and used by thermos-dynamics and hydro-dynamics, do not contain any tie-in start point with molecular structure of actual substance. The concept of ionic/molecular structure and interaction of condensed status are practically not presented in rheology, molecular/kinetic theory of fluids, transfer processes and basic physical/chemical phenomena for condensed media. Such a situation defines the necessity in their dynamic substantiation (Leonov 2014) at the basis of integrated energy approach for the conjugation of equ's at the phase boundaries for structural phenomena in geologically deformed porous media.

At the basis of dynamic conductivity for the conjugated solution of N.-Stokes equ's in view of deformation relaxing (Popkov et al 2014) we propose the innovative model of porous media non-linear consolidation towards the structural/phenomenological and hydro-dynamic phase stress releasing by the saturation of balanced porous skeleton and interaction in scale of geological age. The specific dynamics of time scales is described by the non-linear model that is analogous to quantum one (Datta 2009), Figure 2 with its own energetic spectrum for delaying contacts of stratified micro-structure. The role of gravitational, thermal, capillary concentrating and MAK-waves of the Earth (electro-magnetic, Coriolis and Archimedes forces) is proposed as at the basis of dynamic modification of filtration capacity properties, pseudo-functions of relative phase permeability and constants of piezo- and thermo- conductivity in specific points of phase equilibrium, additional boundary conditions (DHE) for filtration boundaries and stress-deformed status (Jormakka 2010). 
The self-organization of porous space in reservoirs lines with the system of de-compacting channels and traps of crystalline foundation with arrangement and dislocation of fractures, de-compactions at nano- and micro-scaled levels, deformation of structures from "single axis type" (Christianovich 2000) of cavity-slot type of decompressions up to strengthening HC shale traps. For millions of years the harmonic nature of micro-world had completely been reflected at the boundaries of continental and oceanic macro-levels (Figure 1) and this is verifies by the invariant similarity of interference development waves with plunging and uplifting of dichotomous segments of the Earth and bi-quadratic structure of oceans with other planets, like Mars and Mercury.

\section{Theoretical Substantiation of Block-Type Organization of Strata Formation Process}

Scientific discussion of this problem is directly linked with solution of main important theoretical and applied tasks of phase contact in time aspect that is one of the basic problems in mathematical theory of mean-scaling of volumetric mass and wave-type transfer of energy (Jormakka 2010, Kulesh et al 2005). While performing field and experimental studies it was noted that there exist various factors that are dependent on scale factor. These effects are specific both for solid materials with matrix skeleton that have apparent micro-structure and for heterogeneous amorphous and liquid media, including water, oil and gas. The presence of porous media radically changes thermodynamics of hydrocarbon mixes (Maximov 1994). Experiments and theories prove the existence of superficial layers having internal structure.

The change in a degree of media dynamic heterogeneity results in spasmodic qualitative changes, changing their macroscopic structure. The large-scale transitions in heterogeneous media are based on a combination of mathematical theory on averaging "the operators" with high-oscillating properties, asymptotic methods and theory of a filtration (Volodin 1999). The block-type motion of the Earth's lithosphere can serve as one of the geological cases. The most clearly seen situation with lateral motions in formation of structural elements and HC traps of the sedimentary cover is observed while simulating the areas with ancient basement, Figure 4. In connection with planetary stresses they arrange the dynamic structures of stretching and compressing types as well as diagonal shear structures (An 2009).

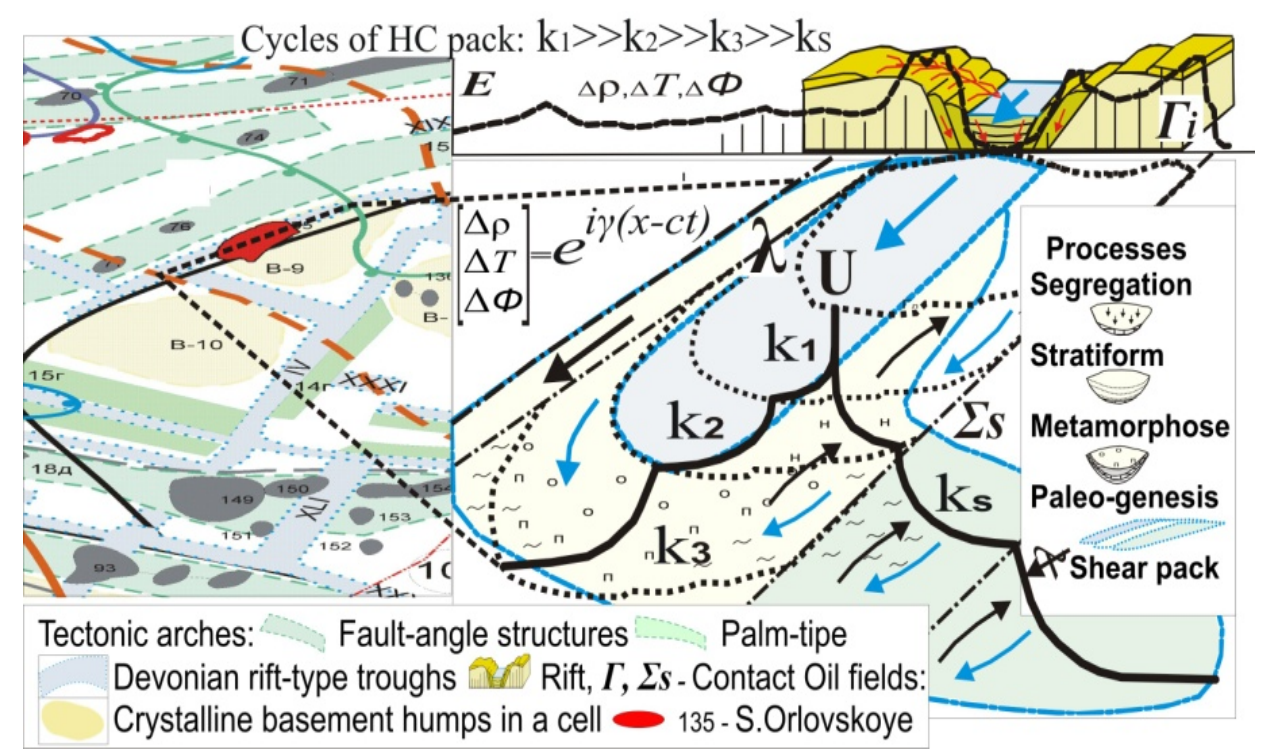

Figure 4. Processes of deflections and uplifted blocks rift-type arrangement Samara Trans-Volga Region

Thus they form the HC deposits with various structural allocations:

- First, the structures connected to erosive surfaces of the basement and with step-type immersing.

- Second, at lateral distribution of stretching stresses, there are finer structures, for which the influence of sedimentary factor (con-sediment processes) is characteristic.

- Third, the compressive stress is clearly seen in the arrangement of neo-tectonic structures.

The urgency of studies with various in scale processes of Paleo \& Neo-tectonics is explained by the fact that 
secondary transformations of rock matrix in particular and $\mathrm{HC}$ systems as a whole, can give up to $80 \%$ of Company production at general tendency of reducing the resource base of "conventional" deposits with "classical" porous type. For example, at the basis of paleo-vulcanological reconstruction of geo-physical well studies and field geological data Dmitrievskiy A. N. has conducted the structural stratification of the reservoirs, and have followed and grounded the rhythmic cycles or sedimentation processes, have illustrated the reservoir and non-reservoir structures at ancient volcanos of Yakutia. These methodological studies were used in various regions of the country: Eastern and Western Siberia, Eastern-European platform (Moscow \& Dnieper-Donets depressions).

The structural changes in porous space are verified by X-ray scanning of dynamic micro-structural contacts done with actual rock cores while they were tested by multi-stage injection with various porous volumes (Roschin et al 2013). Obviously, there are some key moments, like points 1-4, Figure 5c, having spatially distributed skin-layers that are responsible for the individual self-organizing forms with mean phase angle of equilibrium in complexly-organized structure like "de-compacted core- Newtonian viscosity- plastic flow - elastic destruction" inside the gravitational field. There is the channel-core wedge-type angle flow of geological genesis in conditions of phase equilibrium with the arrangement of core/mining structure, e.g. in case with gold miningcharacteristic coral-type intergrowth of massive gold with the angle of $51^{\circ}$, diamond "pipes" or hydro-carbonslatitudinal anticlines, iron-ore deposits, etc. The hierarchy-type organization of microstructures in fractured-porous and capillary-resonant dynamic traps is proved by practical micro-tomography of permeable structures in Earth's geosphere arrangement, in soil and ground, biological and social events (Popkov et al 2014).
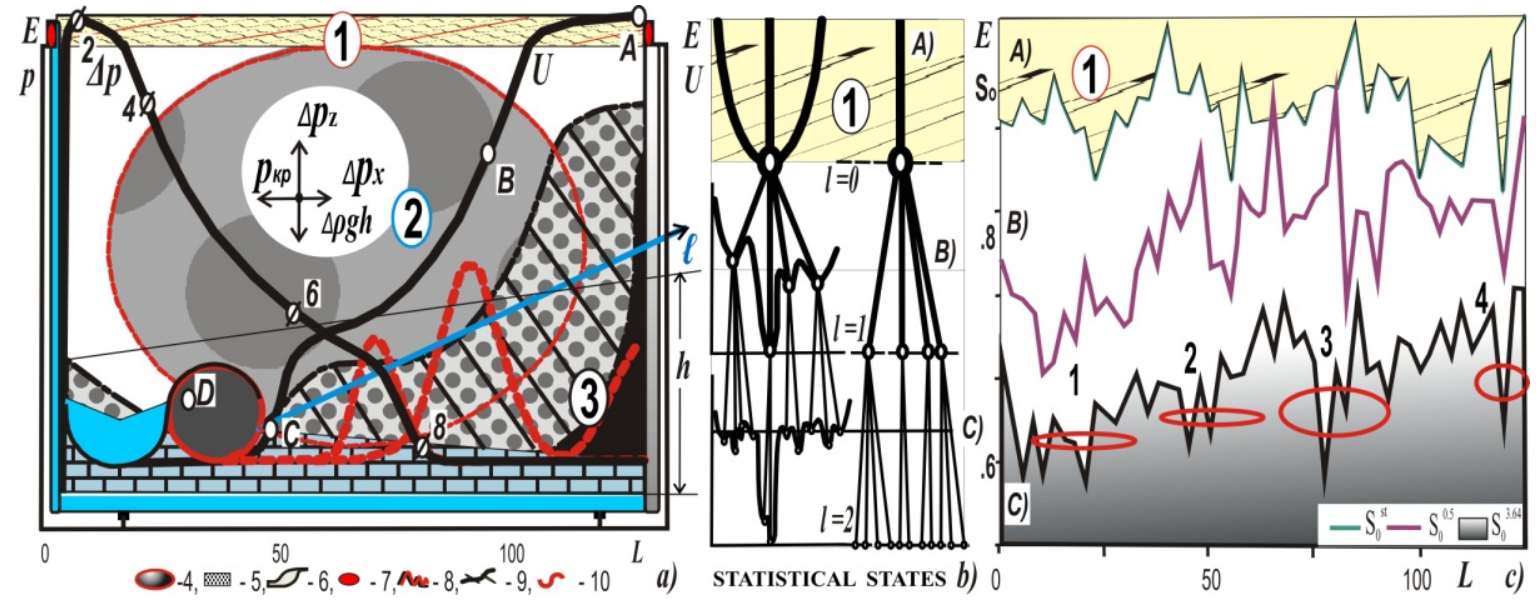

Figure 5(a). Equilibrium in deformations in de-compacted $A$ porous media $B$ and compacted micro-structure $C$ with droplet $D$ detachment; $(b)$ initial boundary conditions $\ell=0$ and further boundary conditions of evolutionary

$\ell=1,2$ for conjugated contact 1 , conductor 2 and matrix 3 , or permeability 4 arrangement at deformation 5 , displacement 6 , perforation 7 , droplet detachment 8 and evolution 9 for porous-cavity wave channel 10 , where $\varnothing(2-8)$ - ID, $h$-dissipative widening; (c) X-ray micro-structure tomography for initial saturation $\mathrm{S}_{0}{ }^{\text {st }}$ and displacement with velocity of $2 \cdot 10^{-6} \mathrm{~m} / \mathrm{s}$ at injection rate of $\mathrm{S}_{0}{ }^{0.5}-0.5, \mathrm{~S}_{0}{ }^{3.64}-3$ volumes and $64 \%$ of pore volume

In the period of continuous media mechanics arrangement the classical scientists paid great attention in studying the deformed microstructure and its effect upon the resistance properties. Hereby we specify the approached of Poisson S. D. (1842), Voigt W. (1887) and brothers Cosserat E. \& F. (1909). The multi-scaled "abnormal rock fluid" behavior and stratification of basic filtration flows is the generalization of a concept of Pollard-Pierson for double porous space, where $\Delta p(t)=\Sigma_{j=1}^{3} a_{j} \exp \left(-\alpha_{j} t\right)$. Here $\alpha_{1}>>\alpha_{2}>>\alpha_{3}$ for fractures, porous reservoir and diffusive matrix respectively. Futher it comes to system-type integratedly conjugated streee interaction for porous space at the basis of a model for generalized visco-elastic feature of micro-world $\Delta p=\Sigma C_{j} \exp \left(-i k_{j} x_{j}\right)+p_{k p}+\rho g h$, where $k_{j}$ - wave number, $p_{k p}$ - capillary pressure, $\rho$ - density of the media, $h-$ thickness of the layer with mean velocity $U$ for outflow as per Darcy, Figure 4.

With stationary movement of the fluid along the current line $l$ the equ'n of micro-structure movement and continuity equ'n a system of differential equ's in partial derivatives with due consideration of structure $h$ scale and relaxation time $\tau$ is as follows (Bykova 2010): 


$$
\begin{gathered}
\rho\left(u_{l} \cdot \partial u_{l} / \partial l+\tau u_{l} \cdot \partial / \partial l\left(u_{l} \cdot \partial u_{l} / \partial l\right)\right)=-\left(\partial p / \partial l+h^{2} / 6 \cdot \partial^{3} p / \partial l^{3}\right), \\
\partial / \partial x_{k}\left(u l_{k}\right)+h^{2} / 6 \cdot \Delta \partial / \partial x_{k}\left(u l_{k}\right)=0
\end{gathered}
$$

and describes the relaxation processes of velocity $u$ and pressure $p$ delays. While resolving N.- Stokes equ's this was achieved by the evolutionary conjugation of boundary conditions at energetic level of interaction phase equilibrium for potential currents $l_{k}$ (Figure 5b). In (Popkov et al 2014) we have got the conjugated solution of inflow in complexly organized deformed reservoir of integral type in quadrature. Potential interaction of generalized function for channel widening $[\mathrm{H}+\mathrm{U}]$ was extended by dissipate part $\Phi$, being the additive energy potential $E$ with local action. The energy of dissipation $\Phi$ is transferred to auto-wave potential energy thus increasing the temperature of chemical reactions, desalination, abnormally high formation pressures and vertical permeability for resonant in-breaks of phase-mineralogical character of interaction - transformation of geological media micro-structure, being the interfacial back-pressure valve and scaled re-distribution of the energy.

\section{Method}

\subsection{Geophysical Conjugation of Geodynamic Tasks for Viscous Flow}

Following the geophysical studies the potential flow of genesis do not possess the absolute symmetry both in permeability and pressure and this is the result of dynamic distribution of velocity and relaxation time extreme gradients for capillary and gravitational forces. By wavy components of porous equilibrium while calculating the potential well production rate Muskat M. (1935) gas provided the substantiation for the flow of poor-permeable dense reservoirs and matrix.

Let's imagine the potential production rate $\mathrm{Q}$ at filtration velocity $U$ of two-layered sphere (fluid + porous skeleton): of permeable layer $H$ (Figure 4) and diffusion-drained sheared zone $h$ with minimum velocity of capillary impregnation $u_{*}$ for micro-world $l_{*}=v / u_{*}$, where $v$ - kinematic viscosity. Let's make conjugation of multi-scaled deformation of micro-world lamella globule and micro-world conductivity for asymmetrically de-compacted (1) porous reservoirs (2) and diffusive impregnation (3) of dense matrix.

\subsection{Energetic Conjugation of Filtration Phase Equilibrium and Porous Deformation. Basic Equations and Boundary Conditions}

The movement of non-compressible fluid with dynamic viscosity $v$ is described by a system of N.-Stokes equ's in Reynolds form (Popkov et al 1998-2014, Nadezhka et al 2010, Yurchenko 2009, Zatsepina et al 2014) for mean values $U$ and pulsing velocities $u$ and continuity

$$
\begin{aligned}
& \partial u_{i} / \partial t+u_{j} u_{i, j}-\left\langle u_{j} u_{i, j}\right\rangle+u_{j} U_{i, j}+U_{j} u_{i, j}=-1 / \rho \partial p / \partial x_{i}+v \Delta u_{i}, \\
& u_{i, i}=0, v U^{\prime}=u_{*}{ }^{2}+\left\langle u_{i} u_{j}\right\rangle .
\end{aligned}
$$

Here $\left\langle u_{j} u_{i, j}=\left(u_{i} u_{i, j}{ }^{*}+u_{i}{ }^{*} u_{i, j}\right) / 4,{ }^{*}-\right.$ is the complex conjugation $i, j=(1-3) \&(x, y, z)$. The initial conditions are $U(\mathrm{x}, 0)=U_{0}(\mathrm{x})$. The equilibrium in porous skeleton is described by the equ'n on movement of viscoelastic media of differential type and generalized form of Hook's Law.

$$
\sigma_{i j}^{q}=\mu^{q}\left(\xi_{i, j}+\xi_{j, i}\right)+\lambda^{q} \delta_{i j} \xi_{i, i}, \sigma_{i j, j}^{q}=\rho^{q} \partial^{2} \xi_{i} / \partial t^{2},
$$

where $\lambda^{q}, \mu^{q}$ - generic parameters of viscoelastic layers $q=1-N, N$ - number of layers; $\sigma, \xi$ - stress and repositioning. At the boundaries of the intermediate layers

$$
\left.\sigma_{i j}{ }^{q-1} n_{j}\right|_{y=h q}=\left.\sigma_{i j}{ }^{q} n_{j}\right|_{y=h q},\left.\quad \xi_{i}^{q-1}\right|_{y=h q}=\left.\xi_{i}^{q}\right|_{y=h q},
$$

where, $n$ is normal. The external layer is fixed $\left(\left.\xi_{i}\right|_{y=0}=0\right)$ or free $\left(\left.\sigma_{i j} n_{j}\right|_{y=0}=0\right)$. Solution at contact $\left.\Sigma\right|_{\mathrm{y}=\mathrm{Yo}_{\mathrm{o}} \text { zone of }}$ conjugation of macro- and micro-world is determined be DHE at

$$
\begin{gathered}
\eta=\left(y-Y_{0}\right) / l^{*}=0: \quad u_{x}=\partial \xi_{x} / \partial t, u_{y}=\partial \xi_{y} / \partial t-\xi_{y} \partial U / \partial \eta, \\
-p+\partial u_{y} / \partial \eta=\sigma_{y y} / \rho u^{* 2}, \quad \partial u_{x} / \partial \eta+\partial u_{y} / \partial x=\sigma_{x y} / \rho u^{* 2} .
\end{gathered}
$$

Here $Y_{0}$ - conjugation contact boundary, and variable figures are dimensionless as per micro-scale $l^{*}=v / u_{*}$ and minimum impregnation velocity $u *$.

DEs $(1,2)$ define the deformation equilibrium condition at the basis of fluid movement equ's in view of inertial elements of viscoelastic skeleton. The generic model of viscoelasticity in conditions of harmonic loading law is defined by the dependencies of shear $\mu$ and elasticity $\lambda$ modules versus frequency $\omega$

$$
\begin{gathered}
\mu(\omega)=\mu_{o}+\sum_{j=1}^{n} \mu_{j}\left(\omega \tau_{j}\right)^{2} /\left(1+\left(\omega \tau_{j}\right)^{2}\right)-i \Sigma_{j=1}^{n} \mu_{j}^{q} \omega \tau_{j} /\left(1+\left(\omega \tau_{j}\right)^{2}\right), \\
\lambda(\omega)=\lambda_{o}-2 / 3\left(\mu(\omega)+\mu_{o}\right),
\end{gathered}
$$


and function of relaxation $\mu(t)=\mu_{o}+\sum_{j=1}^{n} \mu_{j} e^{-t / j j}$, where $\mu_{o}, \lambda_{o}-$ static modules, $\tau_{j}-$ relaxation spectrum.

The system of equ's (1-5) with neglecting the quadratic elements of velocity pulsing allows to make the solution in a form of waves

$$
u_{i}=u_{i}(y) \exp i\left(k_{x} x+k_{z} z-\omega t\right), \quad p=p(y) \exp i\left(k_{x} x+k_{z} z-\omega t\right) .
$$

Considering only attenuating waves by $\eta$, the solution (1) for velocities and pressure in view of deformation is recorded in quadrature as

$$
u_{i}(\eta)=1 / k \int G_{i}(t) \operatorname{sh}[k(\eta-t)] d t+C_{i} e^{-k \eta}, \quad p(\eta)=1 / i k \int G_{4}(t) \operatorname{sh}[i k(\eta-t)] d t+C_{4} e^{-i k \eta} .
$$

Here $G_{x}=\Theta_{x}+u_{y} U_{x}{ }^{\prime}+i k_{x} p, G_{y}=\Theta_{y}+p^{\prime}, G_{z}=\Theta_{z}+i k_{x} p, \Theta_{i}=-i\left(k U_{i}-\omega\right) u_{i}, G_{4}=-i k_{x} U_{x}{ }^{\prime} u_{y}, k^{2}=k_{x}{ }^{2}+k_{z}{ }^{2}$. At the surface with jammed fluid the mean correlation of pulsing pressure and transversal velocity $\langle p \mathrm{v}\rangle=\left(\partial \xi_{1} / \partial t \cdot p^{*}+\partial \xi_{1} / \partial t^{*} \cdot p\right) / 4$ defines the diffusion

$$
\begin{aligned}
\langle p \mathrm{v}\rangle=\operatorname{Re}\left\{1 / i k^{2} \iint G_{2}\left(t_{2}\right) \operatorname{sh}\left(k\left(\eta-t_{2}\right)\right) G_{4}\left(t_{4}\right) \operatorname{sh}\left(i k\left(\eta-t_{4}\right)\right) d t_{2} d t_{4}+C_{2} C_{4} e^{-2 k \eta}+\right. \\
\left.\quad+C_{4} / k \int G_{2}\left(t_{2}\right) \operatorname{sh}\left(k\left(\eta-t_{2}\right)\right) e^{-i k \eta} d t_{2}+C_{2} / i k \int G_{4}\left(t_{4}\right) \operatorname{sh}\left(i k\left(\eta-t_{4}\right)\right) e^{-k \eta} d t_{4}\right\} .
\end{aligned}
$$

The shear stresses coinciding in their direction with pressure wave velocity, i.e. if the torque coincides with flow direction, increase the diffusion and impregnation velocity

$$
\begin{aligned}
& \langle u \mathrm{v}\rangle=\operatorname{Re}\left\{1 / k^{2} \iint G_{I}\left(t_{1}\right) \operatorname{sh}\left(k\left(\eta-t_{1}\right)\right) G_{2}\left(t_{2}\right) \operatorname{sh}\left(k\left(\eta-t_{2}\right)\right) d t_{1} d t_{2}+C_{l} C_{2} e^{-2 k \eta}+\right. \\
+ & \left.C_{2} / k \int G_{I}\left(t_{1}\right) \operatorname{sh}\left(k\left(\eta-t_{1}\right)\right) e^{-k \eta} d t_{1}+C_{1} / k \int G_{2}\left(t_{2}\right) \operatorname{sh}\left(k\left(\eta-t_{2}\right)\right) e^{-k \eta} d t_{2}\right\} .
\end{aligned}
$$

So, the viscoelastic layer of displacement front becomes the re-distributing one. With no shear stresses at the boundary the energy flow $\langle p \mathrm{v}\rangle$ is balanced by dissipation velocity

$$
\Phi=\omega / 4 \int_{0}^{h} i\left\{\left[\lambda^{*}(\omega)-\lambda(\omega)\right] \varepsilon_{i i} \varepsilon^{*}{ }_{i i}+\left[\mu^{*}(\omega)-\mu(\omega)\right] \varepsilon_{i j} \varepsilon^{*}{ }_{i j}\right\} d x,
$$

and factor $Y_{\phi}=\langle p \mathrm{v}\rangle / \Phi=1$ with all the frequencies.

Basing upon the conditions of micro-world filtration conjugation with mean velocity as per Darcy $U=1 / \mathfrak{x} \ln$

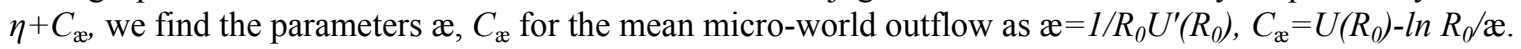

Following the solution of this issue we have obtained the geo-physical conjugation of stress-deformed status with seismic emission diffusive layer, and provide dissipative structure for the generic model of viscoelasticity in dependence of wave number, phase velocity, thickness of the layer and geo-mechanical parameters of geological media.

\section{Basic Frequencies in Resonant Out-flows with Restricted Density and Vertical Anisotropy of Phase Permeability of Deformed Porous Micro-Structure}

While determining the diffusive and dissipative properties in sheared layers of pulsing filtration, let's consider the self-oscillations and behavior of the structure in the area of basic frequencies (Popkov et al 2000-2014, Akbarov et al 2011). Basing upon the root analysis of dispersion equ'n we have considered the character of wave advancement versus the function of losses in material and type of its fixing. We have also recorded the dispersion co-relations of basic oscillation forms for dimensionless viscoelastic cylindrical layer at non-symmetrical boundary conditions. The oscillations of viscoelastic cylindrical layer are described by linearized equ's to preserve the number of movements and by generic Hook's law (2) at boundary conditions (3). This boundary equ'n has the only solution that is dependent on $\omega, \mu_{j}$, $\tau_{j}$. While $\mu_{j}$ is tending to zero we get the task of basic values of ideal elastic body with equalized parameters.

Equ's (2) are presented in the form of Helmholtz and have the solution in a form of longitudinal $\varphi=\varphi(r) \exp (i \gamma z-i \omega t)$ and transversal $\psi=\psi(r) \exp (i \gamma z-i \omega t)$ waves, where $\gamma=\alpha+i \beta-$ is a complex wave number. Substituting solution in (2) in cylindrical system of coordinates we get the system of differential equ's of Bessel's type and obtain the solution as

$$
\begin{gathered}
\varphi\left(z_{\varphi}\right)=c_{l} J_{0}\left(z_{\varphi}\right)+c_{2} Y_{0}\left(z_{\varphi}\right) ; \quad \psi\left(z_{\psi}\right)=c_{3} J_{l}\left(z_{\psi}\right)+c_{4} Y_{l}\left(z_{\psi}\right) . \\
\text { Here } z_{p}=k_{p} r, p=\varphi, \psi, k_{\varphi}=\sqrt{\omega^{2} / a_{\lambda}^{2}-k^{2}}, k_{\phi}=\sqrt{\omega^{2} / a_{\mu}^{2}-k^{2}}, \quad \mathrm{a}_{\lambda}=\sqrt{(\lambda+2 \mu) / \rho,} \quad \mathrm{a}_{\mu}=\sqrt{\mu / \rho} .
\end{gathered}
$$

Satisfying the boundary conditions we get transcendental system of bi-quadratic equ's. The conditions to have non-trivial solution for the system is the zero meaning of determinant. This gives characteristic equ'n of basic wave numbers $\gamma$ and resonant frequencies in viscoelastic layer of geo-physical emission for filtration attenuation and transformation of longitudinal pulses into transversal ones as $\operatorname{det}\{A\}=0$.

Figure 6a presents the values of dimensionless phase velocity $c_{\phi}=c / c_{o}$ (solid line), where $c_{o}=\sqrt{\mu(3 \lambda+2 \mu) /(\lambda+\mu) \rho}$ and attenuation factor $\gamma$ (dotted line) of free layer depending upon the frequency $\omega_{\kappa}=\omega h / c_{o}$. Starting practically 
from zero frequency there appears the bended line spreading at velocity defined by Young's modulus. With low frequencies there appears the bended wave of basic frequency $\omega_{0}$. With frequency growth its phase velocity dashes to velocities on transversal waves. At $\omega_{k}>1$ the porous media generates transversal lines with geo-informative structure, used with seismic studies of lithosphere and HC traps. With basic frequencies there is the sharp reduction in attenuation factors.

Figure $6 \mathrm{~b}$ presents amplitude-frequency spectra obtained at seismic stations 1-CHK, 2-VOS, 3-VRS \& 4-VRH, located within the boundaries of Voronezh crystalline massif at ancient Eastern - European platform. The studies (Nadezhka et al 2010) show that with frequency range between 0.1 and $0.3 \mathrm{~Hz}$ the micro-seismic noises are formed mainly by microseism of de-compacted migration channel incoming from the Atlantic Ocean. Analysis of daily variations in micro-seismic noises within $1-8 \mathrm{~Hz}$ frequency range have shown that correlation of micro-seismic noises there may be motivated by the fact that geological structure and anthropogenic load produce the prevailing effect upon the formation of high-frequency constituent.

Here, in the paper we used also the data for deep-wave channel survey at Lofoten basin obtained in course of XVII Int'l Oceanic Expedition conducted by "Prof. Logachev" research vessel as per UNESCO/MOC program "Floating University (Training through Survey", published in (Yurchenko 2009). Subsea Lafoten basin is located in the northern part of the Norwegian Sea, stretching for more than $200 \mathrm{~km}$ from the mouth of Andoya canyon to the deep-water part of Lofoten basin and is in good correlation with presented model of structural-phenomenological stress release at Middle Oceanic Rift.
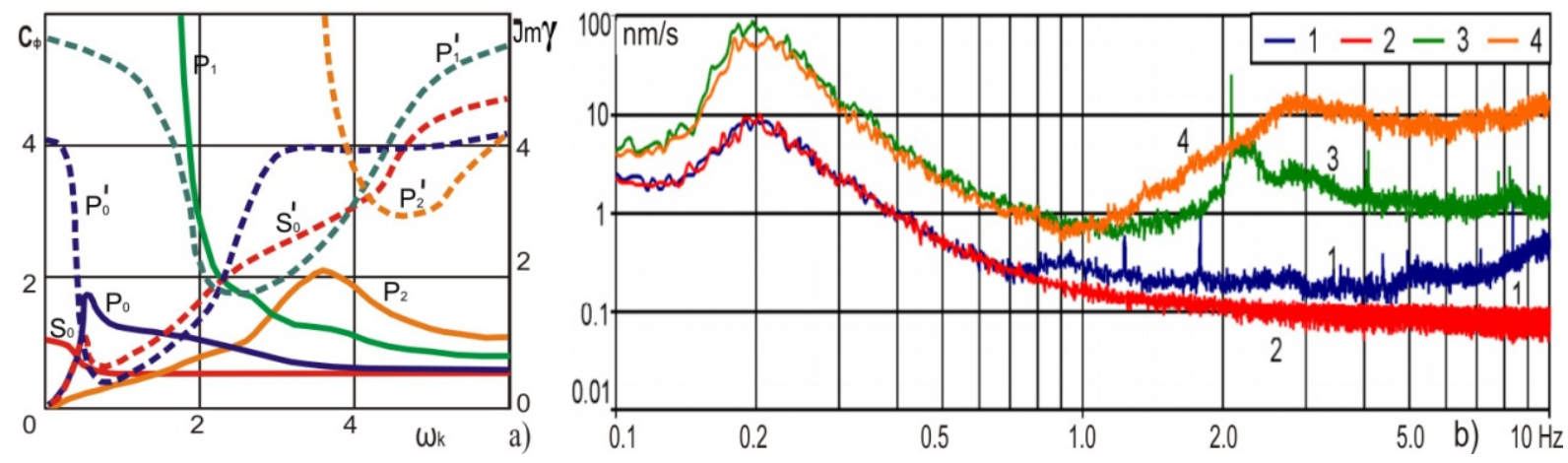

Figure 6(a). Phase velocity and attenuation factor of bended $\mathrm{S}$ and transversal $\mathrm{P}$ waves in viscoelastic free layers; (b) Seismic amplitude-frequency spectrum

The dynamic character of sheared layer is verified by lab experiments to displace the oil from core by water. With velocity increase we observed the growth in Newtonian part of displacement factor. With growth in critical velocity there is the drop in displacement factor, i.e. the completeness of oil recovery factor in majority of the cases depends upon the rate of development.

\section{Interaction Stability in Mean Characteristics of Mass Transfer and Pulsing Deformations}

To understand the thin equilibrium between a viscous layer and non-viscous internal area as well as to confirm a principle of maximal stability for mean characteristics of pulsing boundary layer, we should consider a task to simulate the pulsing current on a viscoelastic porous surface. Thus by an iterative way we take into account the non-linearity of a profile $U(\eta)$ at the basis of the decision in quadrature.

Conditions of the interface which have been written down in the form that doesn't contain the unknown parameters, look like:

$$
\eta \tau^{I}+\tau=1, \eta \tau^{I I}+2 \tau^{I}=0, \eta \tau^{I I I}+3 \tau^{I I}=0, \eta E^{I I}+2 E^{I}=0,
$$

where $\tau=-\rho<\mathrm{uv}>, E=\left(\mathrm{u}^{2}+\mathrm{v}^{2}+w^{2}\right) / 2$. First of them is a condition final normalizing. The requirement to have maximum curvature in a point of interface $\left|E^{I I}(R)\right|$, which provides the maximal stability of the considered profile and is a final precondition in defining the pulsing amplitudes and unknown parameters $k, C_{\mathfrak{x}}, R$.

At each iterative stage the task is to pick up the unknown parameters of $k$ and $c$ for the pulsing movement, so that to make smooth interface in some point $R$ with the profile of mean velocity down to $U^{I V}$ and by mean pulsing energy - down to $E^{I I}$ with asymptotic dependences $U=1 / \propto \ln (\eta)+C_{\alpha}, E=E_{0}(1+B / \eta)$. The parameters of movement 
in a buffer zone are established further by the formulas

$$
a=1 / R_{0} U^{I}(R) ; C_{\mathfrak{x}}=U(R)-\ln (R) / \mathfrak{x} ;-B=R^{2} E^{I}(R) /\left(E(R)+R E^{I}(R)\right) ; E_{0}=R E(R) /(B+R) .
$$

Consequently, the profile of mean velocity is determined for the whole area under the influence of viscoelastic sheared layer in porous micro-structure. The distribution of stresses $\tau / \rho=-<\mathrm{uv}>$ show that non-linearity stabilizes the profiles in respect to small perturbing effect both for rigid and viscoelastic basis. The task becomes correct. Through the application of mean parameter maximum stability principle it was defined that at any phase velocity the stresses have the stability of up to $U \sim 35 \mathrm{u}_{\min }$. The profile conjugation point is in the area of around $\sim 35$ irrespective to boundary conditions and, consequently, the shear layer has practically the same constant thickness. Within the frames of geological age this boundary is drifting and its thickness increases proportionally to accumulation time.

\section{Results}

\subsection{Interconnected Character of SmartWells Multi-scaled Inflow and Lithosphere Defluidization}

The dissipation with filtration in sheared layer plays the role of dynamic distributing valve for the energy mass transfer, Figure 7. With low frequencies $\omega_{k}<1$ there is the bending wave of phase boundary like the membrane for symmetrical ballistic permeability by Darcy with low attenuation factor $Y_{\Phi}<1$. With high frequencies dissipation increases $Y_{\Phi}>1$ and with this the micro-structure becomes an absorbing and compacted one. Energy dissipation gives the increase in temperature of convective diffusion and deformation while shifting HC upwards, i.e. de-compaction of the media. Capillary pressure does not affect the movement, as they are in a state of balance under the Archimedes buoyant force, and horizontal re-positioning as per Stokes are jammed by extreme capillary pressure. The globule is separating at an angle of newly-arranged structure with current wedge of lamination.
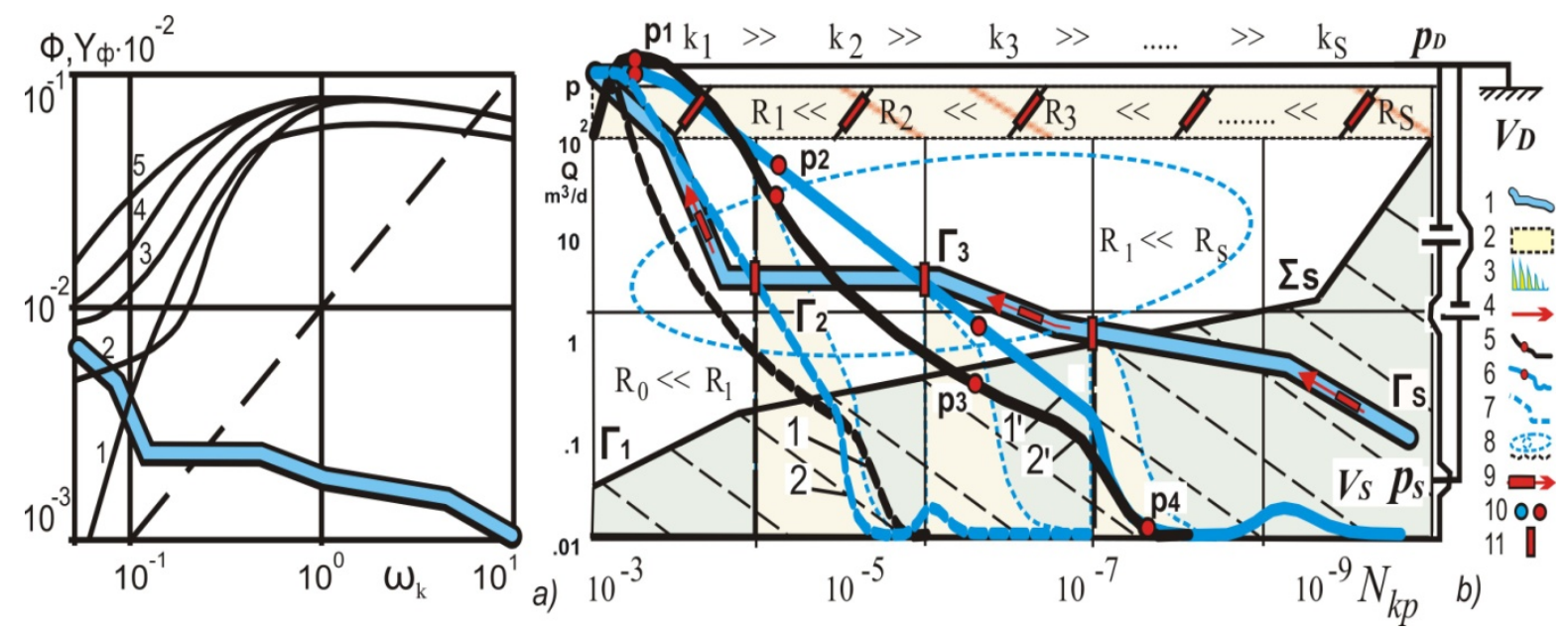

Figure 7(a). Dissipating velocity $\Phi(-)$ and attenuation factor $\mathrm{Y}_{\phi}(-\boldsymbol{C})$ depending upon phase of shearing stress $\tau=1 / \alpha \cdot p \cdot e^{i \varphi \xi}$ at the boundary: $1-\tau=0 ; 2-\alpha=2, \varphi_{\xi}=5 / 12 \pi ; 3-\varphi_{\xi}=1 / 12 \pi ; 4-\varphi_{\xi}=-1 / 12 \pi ; 3-\varphi_{\xi}=-5 / 12 \pi$;

(b) Conjugation of MSHW with $\Sigma$ s contact of porous permeability and compressed matrix

Legend: 1 - MSHW, 2 - de-compaction in de-compacted zones 3, $\Sigma$ s - contact, 4 - unloading trajectory, Ri/ki resistance/permeability, 5-pressure (2, 2'), 6 - inflow Q with packers (1'), 7 - inflow without packer (1), 8 elliptical, parabolic, hyperbolic structures, 9 - resistance, 10 - ID, 11 - packers

Dissipative mass transfer re-distributes the phase flow as per the direction of induced tangential stress $\tau$ with phase $\varphi_{\xi}$ and trajectory $\ell_{\xi}$, Figure 7a. If to consider the perimeter of a round outflow in abnormal filtration with phase angles of weak-energy counter-flow impregnation then pulsing filtration will join-in the main streambed of the flow only in specific phase windows of energy exchange. Invariant similarity in porous micro-structure arrangements, like traps, is observed both at nano- and micro-levels and with macro-scales. Dissipative function is the ruling parameter in hierarchy arrangement of $4 \mathrm{D}$ space of micro-seismic traps: $L_{l}-L_{n}, D_{l}-D_{n}$, where $L, D_{i}-$ the scale of traps and dissipation, $N_{k p}=u / p_{k p}$ - capillary number, Figure $7 \mathrm{~b}$.

For the wells without basic seals in viscoelastic packer's $\Gamma_{\mathrm{i}}$ they appear with single micro-structure of phase velocity distribution, starting from 0 and coming to $2 \pi n$, of scale $L$ for global trap and dissipation $D$. One point in 
applying the energy function of pressure gradient at boundary conditions $\ell=0$ in a wellbore is forming the single elliptical solution for pressure distribution. Viscoelastic packers on multi-segment horizontal wells (MSHW) form basic packing-off and wave generation blocks for excessive pressure with "zero" phase. That's why inside the compacted structure $L$ there form the micro-structures of de-compaction $L_{i}$ and dissipation $D_{i}$ segments of local traps. Elliptical solutions of well segments are tied-in by a single complex-type energetics of smart solution in the area of well drilling, completing, constructing and inflow controlling procedures. This makes the concept of "smart" $\mathrm{HC}$ development, the optimization of well inflow and excessive pressure for enforced turbine effect as with SmartWells (Popkov et al 2014, Zatsepina et al 2014).

Dynamic solution for the channel of de-compacted permeable resonator with porous wave conduit may be considered as MSHW with invariant transformation of geological age scale in geo-dynamic spreading, development of classical HC porous traps and packing-off in matrix lithosphere. From the dissipation viewpoint the dispersed organics in bottom layers of convective-diffusion process of chemical desalination in ancient sedimentary rock of the continents and oceans is up-coming to ancient surface of the Earth and is accumulated in self-organized micro-seismic and tectonic traps. The ancient HC were accumulated by free out-flow of viscoelastic sedimentary rock of the so-called "oil matrix" cycle, complexly organized structure of tangential stresses of Domanik and Bazhenit types. Micro-structure of trap self-organization for oil-gas saturated pools is verified by a distant sounding of electro-magnetic fields with electro-telluric ion flows of Earth's deep genesis. The compacted/de-compacted wave channels of deep genesis were experimentally discovered by electro-sounding of ocean bottom and continental platforms. Located at the crystalline foundation they are associated with geological system of tectonic faults, rifts and de-compacted traps of Melekess depression for the Volga area.

\section{Conclusion}

It was shown that viscous fluids possess contact structural surface with the cells of viscous, plastic and elastic deformation. Due to neo-tectonics of viscous movement there appear energy transformations, arrangement of micro-structure and macro-structure starting from the scale of atomic nano-level up to planetary scale of the Earth.

Global structurally polarized energy basinal-type modeling of the Earth is possible upon the evolutionary conjugation of various types of equ's and additional evolutionary boundary conditions of N.-Stokes equ's.

The hierarchy-type geo-hydro-dynamical barriers are the balanced adiabatic micro-structures of potential "barrier pits" with physical/chemical and geo-physical dissipative boundaries for the development of traps with various relaxation levels.

Then barrier processes are based upon the various physical/chemical nature boundaries for different media in macro- and micro-levels with the arrangement of geo-chemical, structural/geo-hydrodynamic and electro-chemical barriers that play a significant role in trap localization. The petro-graphical barriers due to various physical/mechanical rock properties define the morphology of geological objects and their scale.

Geo-dynamic barriers are the sediment catalysis due to pressure drops in fractures and porous rock. Gravitational/segregation barriers result due to various density, electro-chemical ones - are the sediments at the poles of the electric fields with diffusive and membrane potentials.

The resulted geo-physically conjugated stress deformed status of diffusive layer has shown the seismic emission of dynamic compaction and saturation of micro-structure in porous space.

The mean inflow goes to diffusion impregnation stage and linear outflow - to dissipative auto-wave stage with velocity of $U=35 \mathrm{u}_{\min } \sim 35 \cdot 10^{-7} \mathrm{~m} / \mathrm{s}$ irrespective the type of geological boundary. The de-compacted phase layers move at macroscopic velocity and with low velocity and frequency - the compacted plastic viscoelastic structure of the sheared layer, clay and silt and dolomite substances, that result in boundary swelling, de-compaction of the roof, wedge-type saturation and reservoir stratification.

Analysis of the individual fluctuation forms has shown that for phase-jammed layers there are no actual phase velocities and this gives the process of filtration reduction and attenuation. The same time the free layer of the displacement front always contain the bended wave, and this result in flow disturbance, excessive pressure, wedge-type speed-up of the Newtonian filtrations, fractality of fingering and speedy well water-cutting.

Dissipative properties of porous structure are inter-related with diffusive character of oil-saturation jamming, have maximum value at the boundary of the capillary-porous de-compaction root system. Filtration diffusive properties have optimum character as related to velocity. With velocities higher the critical ones the zone of de-compaction and reservoir destruction is increasing that result in area growth for filtration and absorption 
jamming and $\mathrm{HC}$ sedimentation.

The reservoir structure with double porous space of HC block-type saturation has the character of plastic fracturing, stage-type de-compaction, lamination, arrangement of diffusive resonant barriers in the roof of the trap and convective/diffusion blocks of clay and carbonate-dolomite compacted areas, carbon-mica and high-temperature crystalline carbonate shore deposition.

The root structure of tectonic fracturing, orthogonal to the surface of the least stresses in visco-plastic deformations of genesis de-compaction has the character of elastic break and porous contact with WOC. With no sheared/compacted rim of displacement genesis this refers to porous fractured reservoirs with double permeability in a single layered porous system.

The dissipation of genesis by dispersion and deposition of hydro-carbons takes place along the initially visco-plastic capillary-jammed surface of crystalline water-saturation and has the outlet towards the de-compacted river beds- aqueous traps of the continents. Local segments of genesis elastic mantle is related with the system of tectonic fracturing of traps with porous structure, and indirectly, with WOC. Their global effect goes far beyond the boundaries of the individual contacts between traps/domes, thus forming the maximum amount of $\mathrm{HC}$ traps and generalized outflow for the Middle Oceanic Ridge.

\section{Acknowledgments}

The authors are grateful to Samara State Technical University for financial support of this work as part of the public task of the Ministry of Education and Science of the Russian Federation.

\section{References}

Ablya, E., \& Sokolov, B. (1999). Fluid Dynamical Model of Oil and Gas Formation. Moscow. GEOS.

Akbarov, S. D., Guliev, M. S., \& Kepceler, T. (2011). Propagation of Axisymmetric Waves in an Initially Twisted Circular Compound Bimaterial Cylinder with a Soft Inner and a Stiff Outer Constituents. Mechanics of Composite Materials, 46(6), 627-638. http://dx.doi.org/10.1007/s11029-011-9176-z

An, L. Y. (2009). Paleochannel Sands as Conduits for Hydrocarbon Leakage across Faults: An Example from the Wilmington Oil Field, California. AAPG Bulletin. Amer. Assoc. of Petroleum Geologists, 93(10), 1263-1279. http://dx.doi.org/10.1306/06150909008

Astafiev, V. I., \& Kasatkin, A. E. (2015). Modeling and Numerical Calculation of Piston-type Oil Displacement for Double Periodic Systems of Field Development. Computerized Mechanics of Linear Media, 8(1), 81-92. http://dx.doi.org/10.7242/1999-6691/2015.8.1.7

Ayachi, R. E., Raji, A., Naimi, M., Elharfi, H., \& Hasnaoui. M. (2013). Effect of Sinusoidal Heating on Natural Convection Coupled to Thermal Radiation in a Square Cavity Subjected to Cross Temperature Gradients. Journal of Electronics Cooling and Thermal Control, 3, 7-21. http://dx.doi.org/10.4236/jectc.2013.31002

Berdnikov, K. V., \& Struzhanov, V. V. (2014). Potential Fields with Free Energy at the Stages with Genka Media Compaction and De-compaction at Non-positive Volumetric Deformation. Bulletin of Samara State Technical University, 2(35), 82-88. http://dx.doi.org/10.14498/vsgtu1307

Bouldin, B., Verma, C., Bellaci, I., Black, M., Dyer, S., Algerøy, J., De Oliveira, T., \& Pan, Y. (2014). Prototype Test of an All-Electric Intelligent-Completion System for Extreme-Reservoir-Contact Wells. SPE_Journal. SPE 166507-PA., 19(6), 353-362. http://dx.doi.org/10.2118/166507-PA

Burgers, J. M. (1974). The Nonlinear Diffusion Equation: Asymptotic Solutions and Statistical Problems. Springer Netherlands. http://dx.doi.org/10.1007/978-94-010-1745-9

Bykova, M. I., Verveiko, N. D., Sumets, P. P., \& Shashkina, S. A. (2010). Flow and Deformation of Materials with Micro-structure. Voronezh. VGU.

Christianovich, S. (2000). Fluvial Hydro-dynamics. Filtration Theory. Aero and Gas Dynamics. Mining Engineering. Theory of Plasticity. Energetic. M. MFTI.

Datta, C. (2009). Quantum Transport: From Atom to Transistor. Regular and Chaotic Dynamics. R \& D Center. Institute of computer studies. M. - Izhevsk.

Demyanov, A. Y., Dinariev, O. Yu., \& Evseev, N. V. (2009). Basics of Density Functional Method in Hydro-dynamics. Schlumberger R\&D Center in Moscow. M. PhysMatLit.

Dmitriev, M. N., Dmitriev, N. M., \& Kadette, V. V. (2003). Generalized Darcy Law and Structure of Phase and Relative Phase Permeability for Two-phase Filtration in Anysotropic Porous Media. MZhT. News of RAS. 


\section{2, 136-145. ISSN 0568-5281}

Dmitrievskiy, A. N., Banalyuk, I. E., Donraryan, L. S., Karakin, A. V., \& Poveschenko, Y. A. (2003). Modern Understandings of Hydrocarbon Accumulation in Zones with De-compacted Upper Part of the Crust. Oil and Gas Geology, 1, 2-8.

Garagash, I. A., \& Nikolaevskiy, V. N. (2009). Cosserat Mechanics for Earth Sciences. Computer mechanics for Continuous Medium, 2(4), 44 - 66. Retrieved from http://www.icmm.ru/journal

Gueguen, Y., \& Bouteca, M. (2004). Mechanics of Fluid-Saturated Rocks. International Geophysics. Elsevier Academic Press, 89. ISBN 0-12-305355-2

Jacobs, T. (2014). Studying the Sources of Methane Migration into Groundwater. Journal of Petroleum Technology, 66(12), 42-52.

Jormakka, J. (2010). Solutions to Three-dimensional Navier-Stokes Equations for Incompressible Fluids. Electronic Journal of Differential Equations, 93, 1-14. Retrieved from http://ejde.math.txstate.edu

Kamaletdinov, M. A., Kazantsev, Y. V., \& Kazantseva, T. T. (1981). Nature of Folding. M. Nauka.

Khain, V. I., \& Lomize, V. G. (2005). Geo-tectonics and Basics of Geo-dynamics. M. KDU.

Kulesh, M. A., Matveenko, V. P., Ulitin, M. V., \& Shardakov, I. N. (2008). Analysis of the wave solution of the elastokinetic equations of a Cosserat continuum for the case of bulk plane waves. Journal of Applied Mechanics and Technical Physics, 49(2), 323-329. http://dx.doi.org/10.1007/s10808-008-0044-0

Leonov, V. V. (2014). Electro-Dynamic Phenomena of Physics/Chemistry in Condensed Media. Marginal Tasks and Transient Solutions. Manuscript. Saarbrucken. LAP Lambert Academic Publishing.

Maximov, V. M. (1994). Basics in Hydro-Thermal Dynamics of Reservoir Systems. M. Nedra.

Mironova, E. A., Popkov, V. I., \& Zatsepina, S. V (2013). Eigen Forms in Self-organization of Porous Space and Stress Deformed Status of Earth's Crust. Readings from Ashirov. X Int'l Scientific and Practical Conf. Tuapse, 103-111.

Molofsky, L. J., Connor, J. A., Wylie, A. S., Wagner, T., \& Farhat, S. K. (2013). Evaluation of Methane Sources in Groundwater in Northeastern Pennsylvania. Groundwater, 51(3), 333-349. http://dx.doi.org/10.1111/gwat.12056

Molokovich, Y. (2006). Unbalanced Filtration and Its Application in Oil Field Practical Sphere. Regular and Chaotic Dynamics. R \& D Center Inst. of computer studies. M. - Izhevsk.

Mukhamediev, S. A., Nikitin, L. V., \& Galybin, A. N. (2004). Orientation of Stresses as the Basis for New Objectives in the Area of Continuous Media Mechanics. Models of Continuous Media Mechanics. XVII Int'l School for MCC Models. Papers to Mathematical Center after Lobachevskiy. Kazan, 22, 84-112.

Nadezhka, L. I., Mikhailova, N. N., Nysynbaeev, T. E., \& Pivovarov, S. P. (2010). On Mutual Correlation of Seismic Noise in Various Geological Conditions, Structure, Properties, Dynamics and Minerageny of Lithosphere at Eastern-European Platform. XVI Int'l Conf. Voronezh, 77- 81.

Nikolaevskiy, V. N. (2010). Destruction and Dilatation. Oil and Gas. Vol.1. Regular and Chaotic Dynamics. R \& D Center. Institute of computer studies. M. - Izhevsk.

Popkov, V. I., \& Astafiev, V. I. (2014). Solution of Navier-Stokes Equation Interconnected with Geophysics in Hydrocarbon Search, Exploration and Development. Ciencia'e Tecnica Vitivinicola, 29(7), 298-318. ISSN 0254-0223

Popkov, V. I., Astafiev, V. I., Shakshin, V. P., \& Zatsepina, S. V. (2014). Conjugate solutions of Navier - Stokes equation with deformed pore structure. Bulletin of Mathematical Sciences \& Applications, 3(2), 38-65. Retrieved from http://www.bmsa.us

Popkov, V. I., Shakshin, V. P., \& Voropaev, G. A. (2012). Block-type Self-organization of Earth De-fluidization. Cluster Structure of Time Space. Saarbrucken. Palmarium Academic Publishing. Germany.

Popkov, V. I., Zatsepina, S. V., \& Mironova, E. A. (2014). Reactive-Diffusive Geo-dynamics of Porous Media, Analytics of Navier-Stokes Equation. Euro-Asian Community of Scientists. Modern concepts of scientific studies. IV Int'l Conf., 4(4), 58-61.

Popkov, V. I., Zatsepina, S. V., Khamitov, I. G., Popkova, A. V., \& Mitina, E. V. (2014). Microstructure of Transport in Deformed Porous Media and Energy Conjugated Solution of Navier-Stokes Equation. Practical 
Micro-tomography. III All-Russian Scientific Conf. St.-Petersburg. SPBU, 32-36.

Popkov, V. I., Zatsepina, S. V., Mironova, E. A., \& Dieva, N. N. (2014). Geo-dynamics in Development of Oil and Gas saturated Fields is Frames of Analytical Conjugation of Navier-Stokes Equation and Darcy Law. Math. and Informational Technologies for Oil and Gas Industry. Int'l Conf. named after Chebyshev Russian Math. \& 20 Years of Cooperation between Surgutneftegas \& SAP. Surgut. SurSTU, 64-66.

Popkov, V. I., Zatspina, S. V., Khamitov, I. G., \& Shakshin, V. P. (2013). Method of Energetic Interconnected Junction of Large-Scale Inflow to Smart Wells Constructed in Elaborated Reservoirs SPE Arctic and Extreme Environments Conf. \& Exhibition. SPE 166893, 1434-1457. http://dx.doi.org/10.2118/166893-MS

Radchenko, V. P., Nebogina, E. V., \& Basov, M. V. (2000). Structural and Phenomenon-type Approach Towards Description of Complete Diagram of Viscoelastic Deformation. Bulletin of Higher Institutions. Machinostroeniye, 5-6, 3-13. ISSN 0536-1044

Roschin, P. V., Vaskes-Kardenas, L. K., Tananykhin, D. S., \& Struchkov, I. A. (2013). Lab Studies of Heavy Oil Deposition Process in Carbonate Cores Using X-Ray Tomograph. Readings from Ashirov. X Int'l Scientific $\&$ Practical Conf. Tuapse, 220-224.

Sheretov, Y. V. (2009). Dynamics of Continuous Medium and Space-Time Averaging. Regular and Chaotic Dynamics. R \& D Center Inst. of computer studies. M. - Izhevsk.

Shifrin, E. G., \& Gusev, N. A. (2010). Filtration equations and the Darcy law. Doklady Physics, 55(12), 615-619. http://dx.doi.org/10.1134/S1028335810120074

Vanag, V. K. (2008). Dissipative Structures in Reactive-Diffusion Systems. Regular and Chaotic Dynamics. R \& D Center Institute of computer studies. M. - Izhevsk.

Vernadskiy, V. I. (2001). Chemical Structure of Earth's Biosphere and its Environment. M. Nauka.

Volodin, I. A. (1999). Non-Linear Dynamics of Geological Media. Moscow. GUP VIMI.

Weinberg, A. M. (2009). Computer-aided simulation of transfer processes. Solving of a nonlinear boundaryvalue problems. M. - Jerusalem.

Yurchenko, I. A. (2009). Study of Modern Sedimentary Systems in Deep-water Outflow Cones at the Example of a Subsea Channel at Lofoten Basin, the Norwegian Sea. The Earth: Acute Problems in Geology as Viewed by Young Scientists and Students. Geological Faculty at Lomonosov MSU \& Exxon Mobil Exploration. M. MSU, 161- 164.

Zatsepina, S. V., Mironova, E. A., Popkov, V. I., \& Shakshin, V. P. (2014). Quantum Transport from Transistor to Earth's De-fluidization Channel. Analytics in Solving Navier-Stokes Equation for Porous Media. Math. Physics \& Its Appl. 100 Years Inst. of Steklov RAS \& SamSTU. IV Int'l Conf. Samara, 178-181.

\section{Copyrights}

Copyright for this article is retained by the author(s), with first publication rights granted to the journal.

This is an open-access article distributed under the terms and conditions of the Creative Commons Attribution license (http://creativecommons.org/licenses/by/3.0/). 